\title{
The lactic acid bacteria metabolite phenyllactic acid inhibits both radial growth and sporulation of filamentous fungi
}

\author{
Åsa Svanström', Silvio Boveri ${ }^{1,2}$, Emma Boström and Petter Melin ${ }^{1 *}$
}

\begin{abstract}
Background: Food spoilage caused by molds is a severe problem. In food and feed, e.g. dairy products, sourdough bread and silage, lactic acid bacteria are used as starter cultures. Besides lactic and acetic acid, some strains produce other low molecular weight compounds with antifungal activities. One of these metabolites is phenyllactic acid (PLA), well known for its antifungal effect. The inhibitory effect of PLA has only partially been investigated, and the objective of this study was to elucidate in detail the antifungal properties of PLA.

Results: We investigated the outgrowth of individual conidia from Aspergillus niger, Cladosporium cladosporioides and Penicillium roqueforti, and observed the morphologies of resulting colonies on solid media using different acid concentrations. We found that PLA inhibits molds similar to weak acid preservatives. Furthermore, it has an additional activity: at sub-inhibitory concentrations, fungal colonies displayed slower radial growth and inhibited sporulation. The $L$ isoform of PLA is a more potent inhibitor than the D form. Increased expression of phiA was observed during PLA treatment. This gene was initially identified as being induced by Streptomyces-produced macrolide antibiotics, and is shown to be a structural protein in developed cells. This suggests that PhiA may act as a general stress protectant in fungi.
\end{abstract}

Conclusion: From a food protection perspective, the results of this study support the usage of lactic acid bacteria strains synthesizing PLA as starter cultures in food and feed. Such starter cultures could inhibit spore synthesis, which would be beneficial as many food borne fungi are spread by airborne spores.

Keywords: Aspergillus niger, Conidia, Lactobacillus, Dairy products, Sourdough, phiA

\section{Background}

Spoilage of food and feed due to mold growth and synthesis of mycotoxins is a frequent problem which impacts health, economy and food security [1]. In some products, lactic acid bacteria (LAB) are used as biopreservatives and this may, to varying extents, prevent mold growth. For example, the bacterium Lactobacillus plantarum can be used as starter culture in dairy food products [2] and sourdough bread [3]. Moreover, LAB can be used as silage additives, based on the same principals applied when preserving food [4]. LAB can synthesize, in addition to lactic acid (LA), several low molecular weight molecules with inhibitory properties, e.g. organic acids, cyclic dipeptides, short

\footnotetext{
* Correspondence: petter.melin@slu.se

${ }^{1}$ Uppsala BioCenter, Department of Microbiology, Swedish University of

Agricultural Sciences, P.O. Box 7025, Uppsala SE-750 07, Sweden

Full list of author information is available at the end of the article
}

peptides, hydroxylated fatty acids, and phenyllactic acid (PLA, both the D and L isomers) [5-7].

Many studies have investigated the antifungal activity of LAB strains producing PLA, in culture and model systems [8-10], and at the proteomic level [11]. However, apart from its $\mathrm{pH}$-dependency, little is known about the actual antifungal mechanisms. However, D-PLA is reported to display antibacterial activity [12], and L-PLA is a competitive inhibitor of phenylalanine dehydrogenase in the bacterium Rhodococcus sp. [13]. To optimize the biopreservative effect of LAB, it has been suggested that bacterial strains with the greatest potential antifungal activity could be identified by screening for those producing high levels of PLA [14]. Moreover, PLA synthesis can be induced by adding phenylalanine [15]. In humans, PLA has been reported as an accumulated toxic product in patients suffering from the genetic disease 
phenylketonuria, but it has been shown to be non-toxic in cell lines [16].

One consequence of adding LAB strains as starter cultures is a reduction in $\mathrm{pH}$. It is known that carboxylic acids, e.g. weak acid preservatives, LA, and some other LAB-produced metabolites, including PLA, have antimicrobial activity. According to the weak acid theory, at low $\mathrm{pH}$ the acid is uncharged and can thus cross the hydrophobic plasma membrane. The $\mathrm{pH}$ is higher inside the cell and the acid therefore dissociates, after which the charged anion cannot escape through the plasma membrane, causing stress by the increased activity required to maintain a correct intracellular $\mathrm{pH}$. The trapped anion may additionally damage the cell by disrupting membranes, etc. There are several reports that weak acid preservatives, such as sorbic and benzoic acids, inhibit uptake of nutrients, e.g. amino acids [17-19] and nucleotides [20].

The aim of the present study was: to investigate the inhibitory properties of PLA; to reveal how it acts as a fungal inhibitor; and, to establish the concentrations required to display antifungal activity. We decided to primarily use the common food spoilage fungus Aspergillus niger, because it produces large numbers of asexual spores that are easy to extract, has a sequenced genome, and has been a target mold in other similar studies [9,20-22].

\section{Methods}

\section{Strains and maintenance of fungal cultures}

The fungal species and strains used in this study are listed in Table 1. Cultures were grown on malt extract agar (MEA; Oxoid) at $25^{\circ} \mathrm{C}$. For the uridine auxotrophic A. niger strain AB4.1, all plates were supplemented with $10 \mathrm{mM}$ uridine (Sigma-Aldrich). In all experiments, spores were harvested from 7-day old cultures.

\section{Statistical analysis}

One- and two- way ANOVA with Bonferroni post test (Prism 5, GraphPad Software) were used to analyze statistical differences of the derived results.

\section{Table 1 Fungal strains used in this study}

\begin{tabular}{ll}
\hline Species and strain & Origin/reference \\
\hline Aspergillus niger, N402 & {$[23]$} \\
wildtype & \\
Aspergillus niger, AB4.1 & {$[24]$} \\
Uridine auxotroph & \\
Pencillium roqueforti, J5 & $\begin{array}{l}\text { Dept of Microbiology, Swedish } \\
\text { University of Agricultural Sciences }\end{array}$ \\
Cladosporium cladosporioides J308 & $\begin{array}{l}\text { Dept of Microbiology, Swedish } \\
\text { University of Agricultural Sciences }\end{array}$ \\
\hline
\end{tabular}

Preparation of agar plates supplemented with weak acids The weak acids (all purchased from Sigma-Aldrich), DL-PLA (1:1 ratio), L-PLA acid, D-PLA, and benzoic acid were prepared by mixing each compound with lactic acid (VWR International). The acid mix was subsequently dissolved and sterilized in $1 \mathrm{ml}$ ethanol (99\%) before being added to autoclaved Aspergillus Complete Medium (ACM; per liter: $\mathrm{NaNO}_{3}, 6 \mathrm{~g}$; $\mathrm{KCl}, 0.52$ g; $\mathrm{MgSO}_{4} \cdot 7 \mathrm{H}_{2} \mathrm{O}, 0.52 \mathrm{~g} ; \mathrm{KH}_{2} \mathrm{PO}_{4}, 1.52 \mathrm{~g} ; \mathrm{FeSO}_{4} \cdot 7 \mathrm{H}_{2} \mathrm{O}$, $0.5 \mathrm{mg} ; \mathrm{ZnSO}_{4} \cdot 7 \mathrm{H}_{2} \mathrm{O}, 0.5 \mathrm{mg}$; glucose, 2.0 g; casamino acids, $1.5 \mathrm{~g}$; bactopeptone, $2.0 \mathrm{~g}$; and yeast extract, $1.5 \mathrm{~g}$ ). Immediately before pouring, the nutrient solution was mixed with autoclaved agar solution kept at $50^{\circ} \mathrm{C}$, to give a final agar concentration of $1.5 \% \mathrm{w} / \mathrm{v}$. For each acid blend, a total nutrient-agar solution of $100 \mathrm{ml}$ was prepared and $80 \mathrm{ml}$ were evenly distributed among four $90 \mathrm{~mm}$ petri dishes, with the remaining $20 \mathrm{ml}$ used for $\mathrm{pH}$ measurement. For most assays, the total acid concentration was $150 \mathrm{mM}(\mathrm{pH}=2.9)$. A. niger N402 DL-PLA was also tested on plates with $150 \mathrm{mM}$ acid at $\mathrm{pH} 4.4$ (adjusted with $\mathrm{KOH}$ prior to adding the agar). The acid blends comprised mixtures of LA and PLA, the latter typically added in increasing increments of $15 \mathrm{mM}$, i.e. starting with $15 \mathrm{mM}$ PLA and $135 \mathrm{mM} \mathrm{LA}$, up to $90 \mathrm{mM}$ PLA and $60 \mathrm{mM}$ LA. However, for Penicillium roqueforti, PLA was mixed in increments of $10 \mathrm{mM}$, up to $40 \mathrm{mM}$ PLA. For benzoic acid, the first blend comprised $0.75 \mathrm{mM} / 149.25 \mathrm{mM} \mathrm{LA}$, with increasing increments of $0.25 \mathrm{mM}$ up to $3.0 \mathrm{mM}$. For A. niger AB4.1 and Cladosporium cladosporioides J308, the total acid concentration of LA and PLA was set at $100 \mathrm{mM}$ ( $\mathrm{pH}=3.5)$, with increments of $10 \mathrm{mM}$ PLA up to $40 \mathrm{mM}$.

\section{Spore preparation, colony morphologies and estimations} of minimal inhibitory concentrations

Spores were harvested by adding $0.15 \mathrm{M} \mathrm{NaCl}$ containing $0.2 \%$ of Tween 80 to fungal colonies on agar plates. Next, the conidial suspensions were diluted in $0.15 \mathrm{M} \mathrm{NaCl}$, counted in a Bürker chamber, and finally adjusted to 250 spores in $1 \mathrm{ml} 0.15 \mathrm{M} \mathrm{NaCl} .100 \mu \mathrm{l}$ of spore suspension, i.e. 25 spores was evenly spread on ACM agar plates (in four replicates) containing different acid blends. Spores were also spread on ACM control plates containing either $100 \mathrm{mM}$ or $150 \mathrm{mM} \mathrm{LA}$, and on MEA plates.

The inoculated agar plates were incubated at $25^{\circ} \mathrm{C}$ for 14 days. The colonies were examined and counted after 3,7 and 14 days to estimate the minimal inhibitory concentrations (MIC), here defined as when no colonies were noted during a thorough macroscopic observation. Any differences in colony growth and morphologies were also visually observed and recorded.

\section{Radial growth and spore count}

The radial growth of single $A$. niger N402 colonies was measured on ACM plates containing $15 \mathrm{mM}$ DL-PLA/ 
135 mM LA and on ACM control plates with 150 mM LA, centrally inoculated with approximately 10 spores and incubated at $25^{\circ} \mathrm{C}$ for 3 and 7 days. The sporulation densities on the same plates were estimated by removing a circular area of $95 \mathrm{~mm}^{2}$ (11 mm diameter) from the center of the colonies. Samples were vortexed with $2 \mathrm{ml}$ of $0.01 \%$ Tween 80 and 10 glass beads ( $2 \mathrm{~mm}$ in diameter) for 10 min before counting the spore concentration in a Bürker chamber. Measurements of radial growth and sporulation were conducted on three biological replicates with three technical replicates each.

\section{RNA extraction, CDNA synthesis and real-time PCR}

To extract total RNA, fungal spores were inoculated on plates covered with membrane filters (mixed cellulose esters, Metricel ${ }^{\oplus}$, Pall Corporation, Michigan). After incubation for 3 and 7 days, the filters with growing colonies were transferred to mortars containing liquid nitrogen and ground to a fine powder with the pestle. Further extraction, cDNA synthesis, quantitative real-time PCR and calculations were performed essentially as described previously [25]. The real-time PCR protocol comprised the following steps: initial denaturation and enzyme activation $95^{\circ} \mathrm{C} 30 \mathrm{~s} ; 40$ cycles of $95^{\circ} \mathrm{C} 2 \mathrm{~s}$ and 56 $60^{\circ} \mathrm{C}$ for $8 \mathrm{~s}$; plate read, and finally, melt curve analysis starting at $65^{\circ} \mathrm{C}$ and ending at $95^{\circ} \mathrm{C}$. The primers used are listed in Table 2. All results were calculated from three biological replicates with three technical replicates.

\section{Results}

Assay setup and growth inhibiting properties of phenyllactic acid

In preliminary experiments examining the inhibitory effect of PLA at sublethal concentrations on wildtype $A$. niger

Table 2 Primers used in this study

\begin{tabular}{|c|c|c|}
\hline $\begin{array}{l}\text { Primer } \\
\text { name }\end{array}$ & Sequence $5^{\prime}-3^{\prime}$ & $\begin{array}{l}\text { Purpose/accession } \\
\text { number }\end{array}$ \\
\hline $\mathrm{T} 18 \mathrm{r}$ & TागाताातााताTVN & cDNA synthesis \\
\hline actF & TCGTGACCTGACGGATTACCTC & qPCR actin \\
\hline actR & TGGAAGAAGGAGCAAGAGCAGTG & ANI_1_106134 \\
\hline treBF & TGGACACTTACCTCTGGGATGAAG & $\mathrm{qPCR}$ treB \\
\hline treBR & GCTGATGGGCATTGAGTATTTCC & ANI_1_1258014 \\
\hline tppAF & TTGAAGACACCGTTGGGAAGAG & qPCR tppA \\
\hline tppAR & GGAGCAAAAGATGAACTCAGGAGC & ANI_1_1432094 \\
\hline phiAF & AGTTCGGTGGAAAGGACCTG & qPCR phiA \\
\hline phiAR & ATACCCTCACAGCCCTCGTTGTAG & ANI_1_280124 \\
\hline brlAF & TGGGCAATGTGATACCTTG & qPCR brlA \\
\hline brlAR & AATGAGCAGGAACGCACTGGAG & ANI_1_2984014 \\
\hline vOsAF & TACGGAATCCCATCCTCCAC & qPCR vOsA \\
\hline VOSAR & GTCTGGTATTGCGAACGAG & ANI_1_952184 \\
\hline
\end{tabular}

spores, we found that fungal colonies grew more slowly and sporulation was inhibited (data not shown). However, increased concentrations of PLA in the culture medium resulted in a decreased $\mathrm{pH}$. Accurately adjusting the $\mathrm{pH}$ with $\mathrm{HCl}$ to be equivalent to the highest PLA concentration tested was not possible since agar is rapidly destroyed if kept at acidic $\mathrm{pH}$ over a period of time and adding $\mathrm{HCl}$ just before pouring the plates resulted in too large variation in the final $\mathrm{pH}$-value. Therefore, to obtain a constant $\mathrm{pH}$ in the following experiments, we used a mix of PLA with the potentially less inhibitory LA. Total PLA/LA concentrations of $100 \mathrm{mM}$ and $150 \mathrm{mM}$ resulted in $\mathrm{pH}$ values of $3.5(+/-0.05)$ and $2.9(+/-0.05)$, respectively, and there were no measurable differences when changing the relative concentrations of the two acids, despite the slight difference in their $\mathrm{p} K_{\mathrm{a}}$ values (3.86 for LA and 3.72 for L-PLA).

Using this experimental approach, we found that the growth and colony morphologies on control plates with $150 \mathrm{mM}$ LA were generally identical to controls on MEA (Figure 1A-B). At pH 4.4 and a DL-PLA/LA concentration of $90 / 60 \mathrm{mM}$, the radial growth was similar to the two controls but sporulation was delayed in some colonies (Figure 1C, assayed visually). In contrast, the same acid blend at pH 2.9 (namely DL-PLA at $60 \mathrm{mM}$ ) was sufficient to totally repress growth of $A$. niger, i.e. the MIC was $60 \mathrm{mM}$. After 3 days incubation at $25^{\circ} \mathrm{C}$ with $15 \mathrm{mM}$ of DL-PLA, radial growth was affected, and this was even more pronounced after 7 days when an apparent reduction in sporulation was also observed (Figure 2A and B, numerically assayed). Higher levels of sub-inhibitory concentrations, i.e. 30 and $45 \mathrm{mM}$ of DL-PLA affected the radial growth and reduced the sporulation in a concentration dependent manner (Figure 3A, assayed visually). After 14 days, the MIC had not changed but sporulation had been initiated at lower acid concentrations. However, at $45 \mathrm{mM}$ DL-PLA, sporulation was still limited and the colony sizes were smaller compared to the control (Figure 3B, assayed visually). Sub-inhibitory concentration of DL-PLA did not appear to decrease the number of colonies formed (Figure 3A). Note that morphologies of the controls were always observed after 3 days of incubation, as at later stages the plates were overgrown.

In order to check whether the two isoforms of PLA, i.e. the $\mathrm{L}$ and $\mathrm{D}$ forms, differed in activity, we repeated the test but using the two forms separately. The MIC values after both 7 and 14 days were as follows: $60 \mathrm{mM}$ for D-PLA and $45 \mathrm{mM}$ for L-PLA. When we examined the colonies under a stereomicroscope after 7 days of incubation on DL and L-PLA, we could only observe undifferentiated hyphae (Figure 3A,C). A few individual conidiophores could be observed on these colonies after 14 days, and on D-PLA plates after just 7 days (Figure 3B,D). 

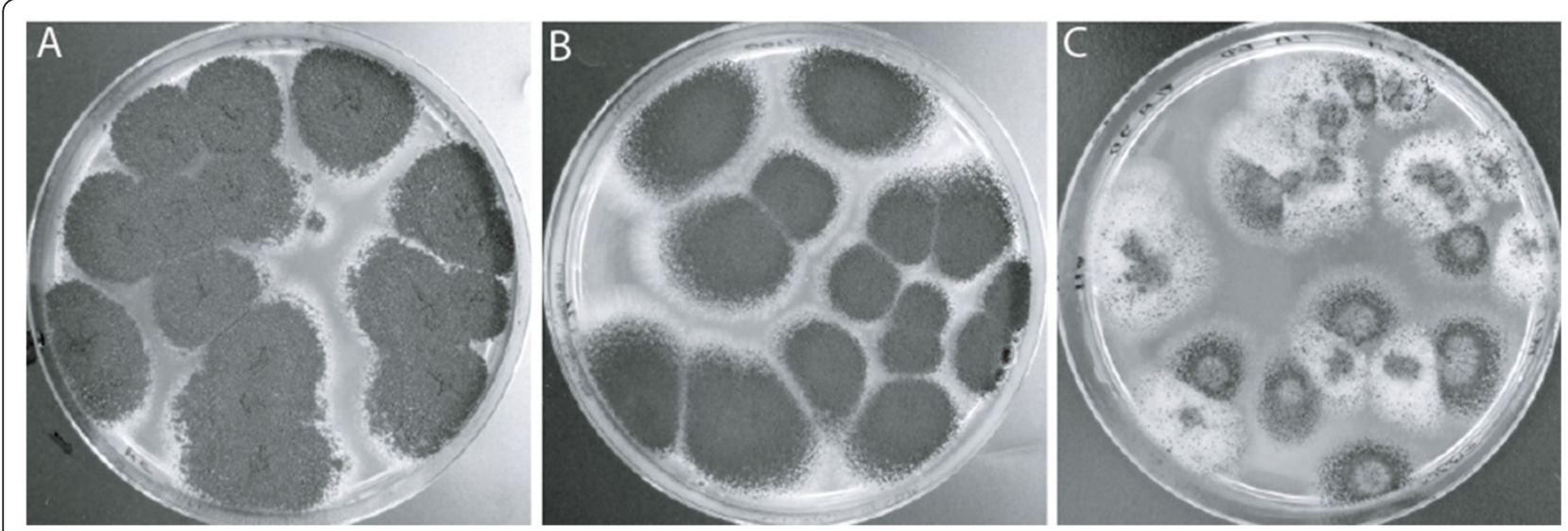

Figure 1 Morphology of $\boldsymbol{A}$. niger on control plates and with PLA. Colonies grown 7 days on $90 \mathrm{~mm}$ petri dishes. Each plate was spread with approximately 25 conidia. Control on MEA (A). Control on 150 mM LA, pH 2.9 (B). Spores assayed with 90 mM PLA and 60 mM LA at pH 4.4 (C).

\section{Shared features between phenyllactic acid and weak acid preservatives}

It could be argued that traditional weak acid preservatives in combination with LA affect colony growth and sporulation in a similar manner to PLA. To test this, we subjected A. niger wildtype N402 spores to benzoic acid (BA) that similar to PLA also contains a phenyl group. After 7 days of incubation, the MIC value of BA was $1.0 \mathrm{mM}$ and after 14 days it was $1.5 \mathrm{mM}$. In contrast to PLA, we did not observe any changes in growth rate or sporulation of the growing Colonies. Moreover, besides being effective at much lower concentrations compared to PLA, BA reduced the total number of colonies even at sub-inhibitory concentrations (i.e. below the MIC; Figure 4A-B), indicating a fungicidal activity.

It has previously been reported that uridine and adenine auxotrophs of Aspergillus niger were oversensitive to all tested weak acids, and that inhibited nutrient uptake was the likely mechanism [20]. To determine if PLA has this activity, we repeated our assay with PLA, but replaced the wildtype with a uridine auxotroph A. niger strain (AB4.1). The mutant was very sensitive and did not grow on the 150 mM LA control plates; therefore, we instead used a total acid concentration of $100 \mathrm{mM}$. In the control, the mutant strain grew similar to the wildtype controls, but addition of DL-PLA (10 mM PLA and 90 mM LA) resulted in fewer spores germinating compared to the control (Figure 4A,C-D), although the colony morphologies mostly resembled the control (Figure 4C-D). The MIC was estimated to be $15 \mathrm{mM}$ in presence of $85 \mathrm{mM}$ LA. Under these conditions, the wildtype strain N402 was unaffected both in number of viable colonies and colony morphology (data not shown).

\section{Gene expressions of Aspergillus niger assayed with phenyllactic acid}

To elucidate the effects of PLA compared to LA on sporulation, the expression of five genes known to be important for asexual reproduction in Aspergilli were examined. These genes were: phiA, induced by V-ATPase inhibitors [26-28] and essential for phialide development [29]; tppA, the $A$. niger tps 2 ortholog encoding trehalose phosphate phosphatase [30], also essential for normal sporulation in Aspergullus fumigatus [31]; vosA, which is important for conidial maturation and also functions as a negative
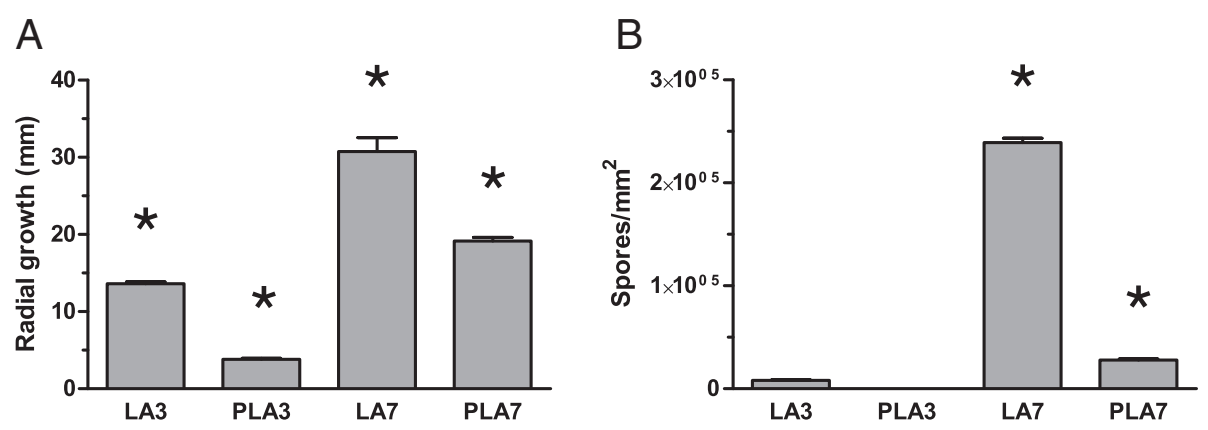

Figure 2 Outgrowth and sporulation of single $\boldsymbol{A}$. niger colonies assayed with PLA. Colony diameter in mm with $150 \mathrm{mM} L A$ and 15/135 mM PLA/LA after 3 and 7 days (A) and spore densities of the same colonies expressed as spores per square mm (B). Error bars show standard error of the mean. The asterisks indicate values that were significantly different to all other values $(p<0.05)$. 

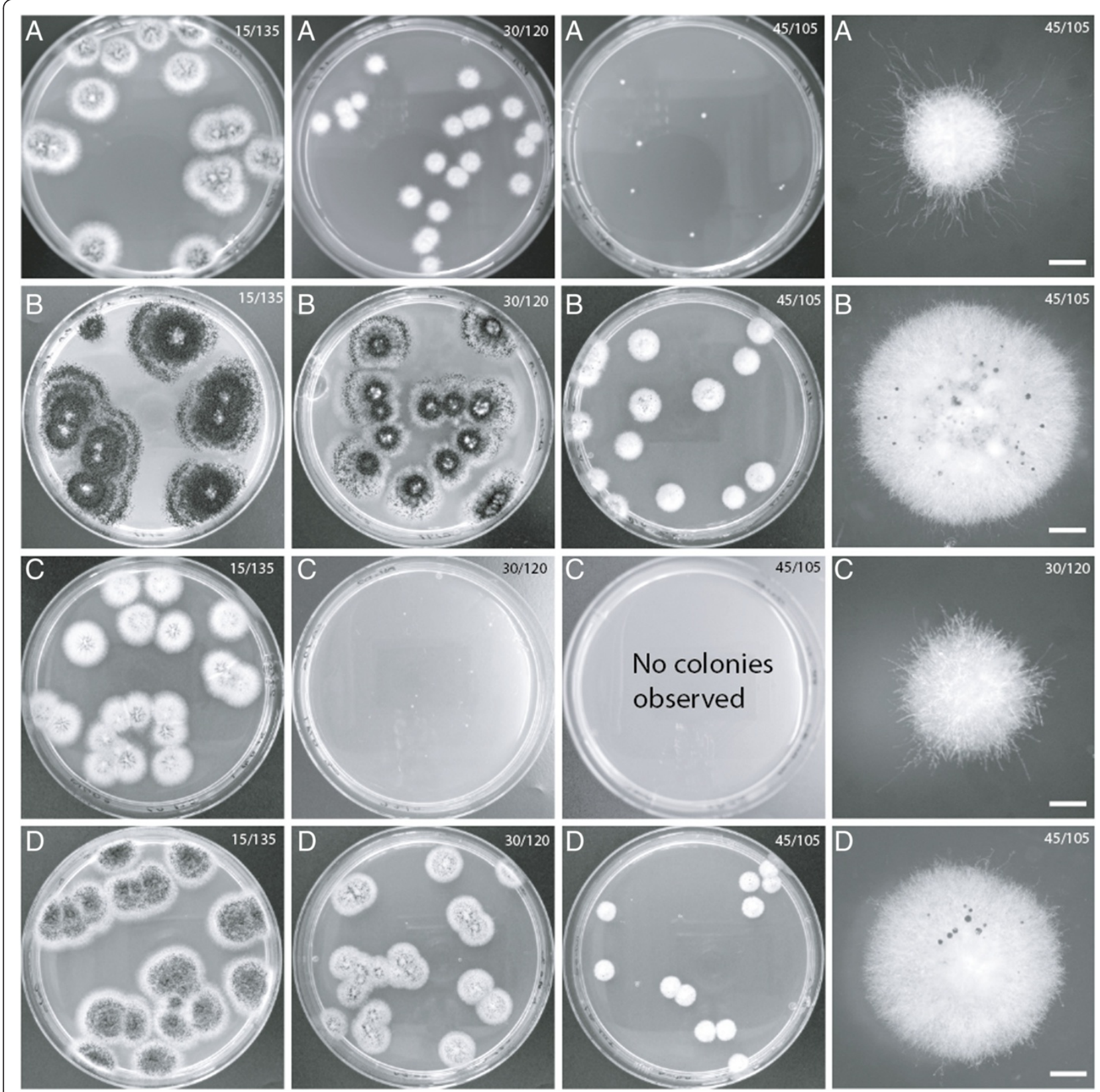

Figure 3 A. niger colonies grown at different concentrations of PLA and LA. Pictures show $90 \mathrm{~mm}$ petri dishes. Concentrations of PLA and $\mathrm{LA}$ in $\mathrm{mM}$ are given at the top right corner of each subfigure. Scale bars where present $=1 \mathrm{~mm}$. Photos taken after 7 days (A) and after 14 days (B) of spores assayed with a 1:1 DL-PLA mixture, L-PLA after 7 days where no growth was observed at $45 \mathrm{mM}$ (C), and D-PLA after 7 days (D).

feed-back regulator of sporulation [32]; treB, the gene encoding for intracellular trehalase, which has been shown to be involved in spore production and viability [25]; and $\operatorname{brl}$, which regulates conidiophore stipe development [33]. All tested genes were expressed under all conditions, except for brlA in 3-day PLA cultures (Figure 5). The expression of phiA was up-regulated 9 fold in PLA 7-day cultures compared with the LA control after 7 days, and compared with both conditions after 3 days. The expression of $\operatorname{vos} A$ was up-regulated 6-fold in the 7-day cultures compared with the 3-day cultures for both LA and PLA. There were no significant differences in gene expression among the other genes under any condition.

\section{Inhibitory effects of phenyllactic acid tested on two other} filamentous fungi

To widen the study and to determine if the observed effect in $A$. niger is common to other fungi, we repeated the test with DL-PLA on two other spoilage molds: Penicillium 

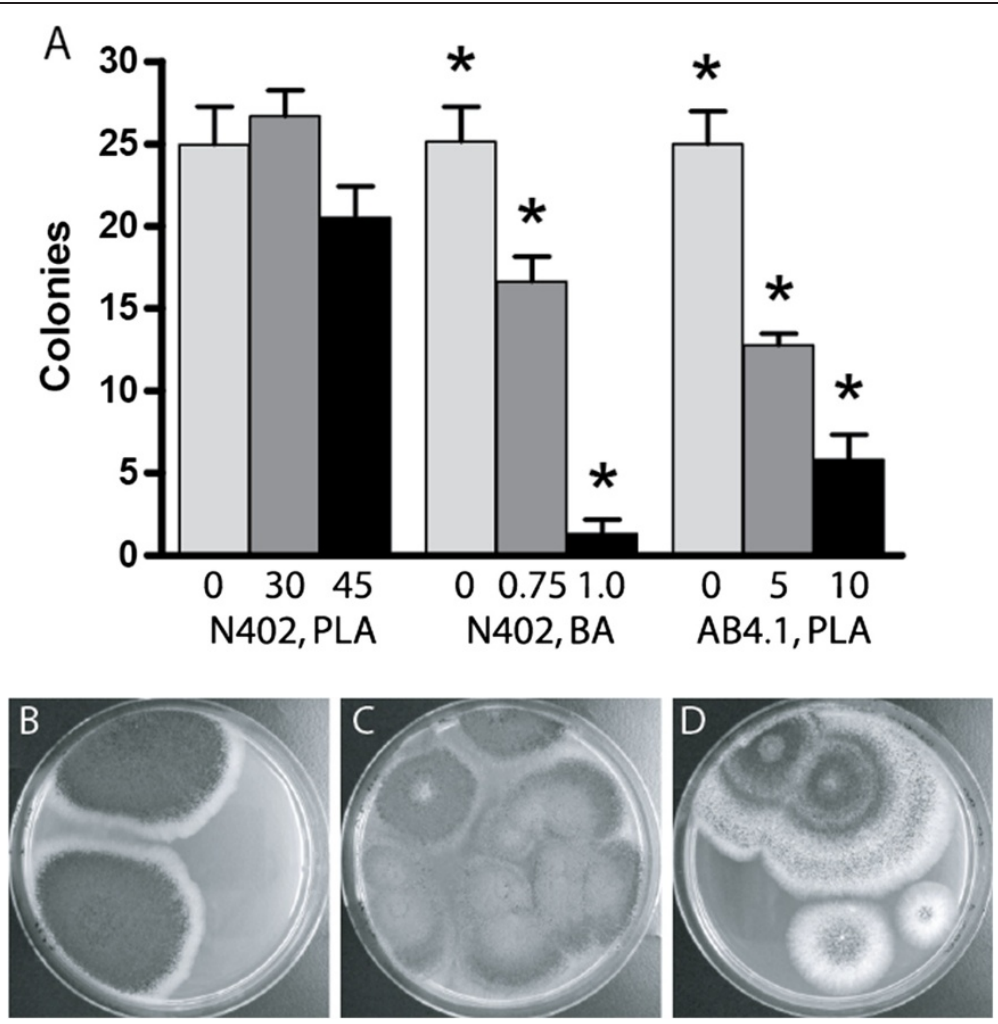

Figure 4 Colony survival and morphology of $A$. niger wildtype and AB4.1 assayed with PLA and BA. The graph (A) shows colony survival under acid stress, the concentration (mM) of DL-PLA or BA is given on the $x$-axis. For wildtype strain N402, LA was added to a total acid concentration of $150 \mathrm{mM}$, and for AB4.1, LA was added to $100 \mathrm{mM}$. The $y$-axis represents the average number of observed colonies (from 4 replicates, normalized to an average of 25 colonies in the controls) after 3 days (controls) and 14 days (assayed samples). Error bar show standard error of the mean. The asterisks indicate colony numbers that were significantly different to all others in the same group $(p<0.01)$. Morphologies of N402 colonies incubated for 14 days on $90 \mathrm{~mm}$ petri dishes assayed with $1 \mathrm{mM}$ benzoic acid and $149 \mathrm{mM}$ LA (B), the uridine auxotroph strain AB4.1 on control plate with $100 \mathrm{mM} \mathrm{LA}$ (C), and AB4.1 assayed with $10 \mathrm{mM} \mathrm{DL-PLA}$ and $90 \mathrm{mM} \mathrm{LA}$ (D).

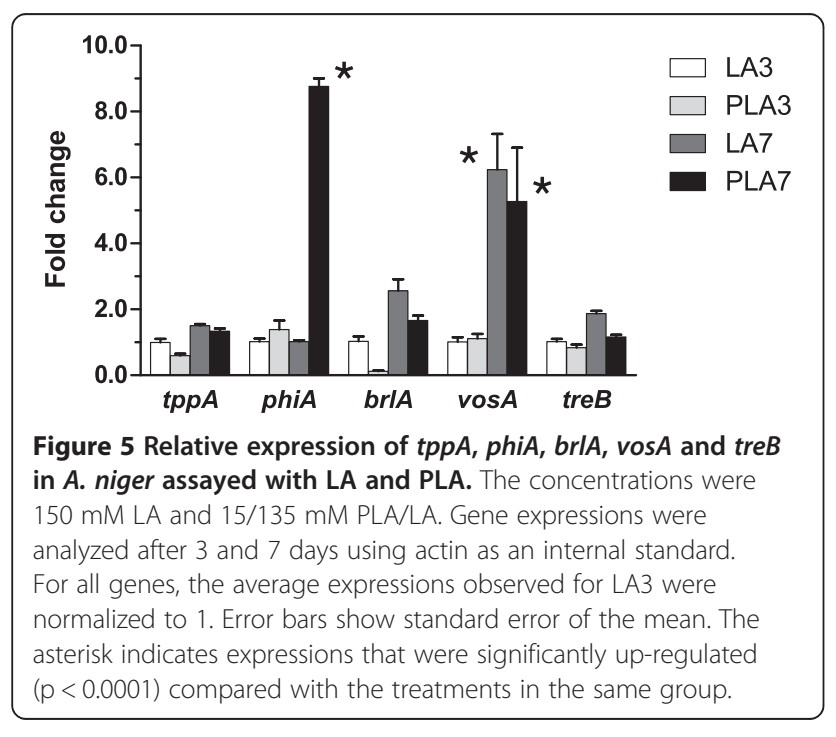

roqueforti, which is a frequent spoilage organism on cheeses, bread, stored cereal grains, etc.; and Cladosporium cladosporioides, which often spoils fruits and wheat. Similar to $A$. niger, both these species produce large numbers of spores enabling the same experimental approach. The MIC for DL-PLA on P. roqueforti was estimated to be $25 \mathrm{mM}$, and at $20 \mathrm{mM}$, a similar morphology to that of $A$. niger at sub-inhibitory concentrations of PLA was observed, namely restricted growth and poor conidiation (Figure 6). C. cladosporioides was not able to grow on the control plates with $150 \mathrm{mM}$ LA at $\mathrm{pH}$ 2.9. Therefore, we modified the conditions for this species to those used for AB4.1, i.e. a total acid concentration of $100 \mathrm{mM}$ and $\mathrm{pH}$ 3.5. Under these conditions, we calculated the MIC to be $30 \mathrm{mM}$, and at $20 \mathrm{mM}$, radial growth was reduced. However, in this fungus the sporulation was unaffected compared to the control (Figure 6).

\section{Discussion}

In this study we have revealed previously unknown inhibitory modes of action of the Lactobacillus-produced 

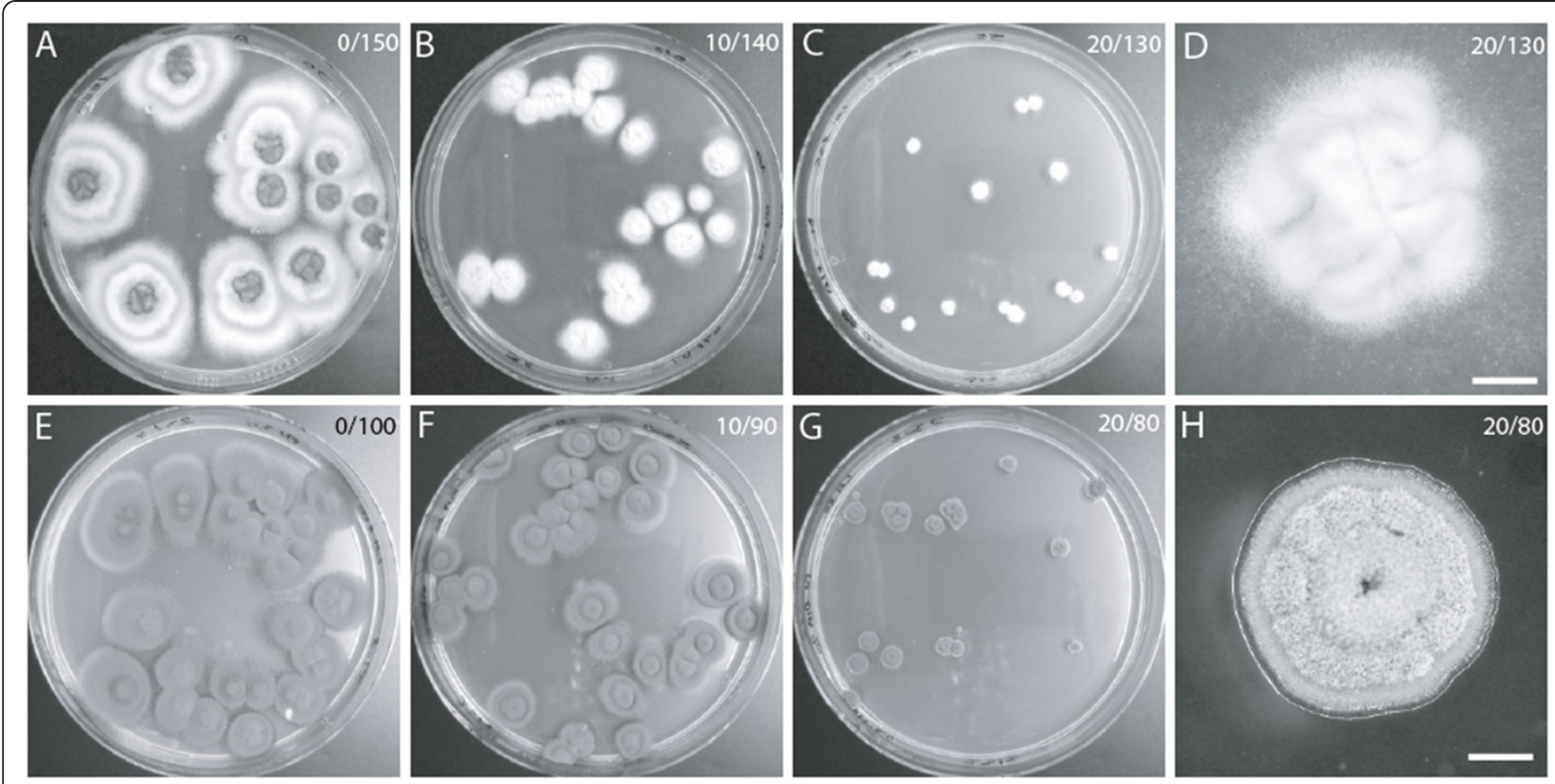

Figure 6 Two additional fungi assayed with DL-PLA. Concentrations of PLA and LA in mM are given at the top right corner of each subfigure. Scale bars when present $=1 \mathrm{~mm}$. P. roqueforti (A-D), $90 \mathrm{~mm}$ petri dishes $(\mathbf{A}-\mathbf{C})$ and a single colony (D). Sporulation was only observed in the $L A$ control (A). C. cladosporioides (E-H), $90 \mathrm{~mm}$ petri dishes (E-G) and a single colony (H).

metabolite PLA. We found that PLA at concentrations close to the MICs reduced the growth of all three tested molds and inhibited sporulation of $A$. niger and P. roqueforti. Notably, Cladosporium does not produce the same advanced conidia-producing structures, conidiophores, as Aspergillus and Penicillium [34]. Consequently, the results indicate that PLA inhibits hyphal differentiation rather than conidial syntheses per se; this was also supported by the gene expression results, discussed in detail below. This inhibitory mode was not observed for the structurally-similar weak acid preservative, benzoic acid. In contrast, benzoic acid was a stronger fungal inhibitor (with lower MIC) and displayed a higher level of fungicidal activity compared to PLA. However, the apparent lack of fungicidal activity may not be a problem, as in dairy products, and silage for animal feed, PLA-producing bacteria are viable and may be able to synthesize PLA constitutively, providing a continuous protection from spoilage fungi even if the compound is consumed or degraded by the spoilage mold.

Our results from the assay of the uridine auxotroph AB4.1 with PLA - the MIC value was lower and fewer spores germinated - indicate that at any concentration, PLA acts in a similar manner to other weak acids by inhibiting nutrient uptake [20]. Furthermore, as it is a rather large molecule, the inhibitory activity is greater compared to that of smaller molecules such as LA. At higher concentrations, PLA starts to influence the fungus by reducing growth and sporulation. In the oversensitive
AB4.1 mutant, only the weak acid mode is active, as the spores were totally prevented from germination at a PLA concentration insufficient to cause the specific morphological changes. The hypothesis that the PLA main mode of function is the same as for other weak acids is further supported by the assay at high $\mathrm{pH}$. When the external $\mathrm{pH}$ is low, weak acids exist in their undissociated form, and being lipophilic, they are able to cross the fungal plasma membrane [21]. At $\mathrm{pH} 4.4$, colony sizes or number were not reduced in $A$. niger cultures at $90 \mathrm{mM}$ PLA compared to the control, indicating that the function of PLA is $\mathrm{pH}$-dependent in a weak acid manner.

Minimal inhibitory concentrations of PLA have been reported previously $[9,10]$. However, in this study we could also show that L-PLA is the most potent inhibitor, although D-PLA has a similar, but lower, activity. This observed difference between the isoforms contradicts previous findings [9]. However, besides estimating MICvalues with smaller increments in concentration, we monitored the outgrowth of individual conidia instead of observing growth in liquid media with high spore inoculums. Our approach has particular relevance in the food spoilage context, as mold spoilage often starts when one single airborne conidium lands on the surface and germinates. It has been reported that sub-inhibitory concentrations of preservatives can induce mycotoxin production [35], and this could be an argument against the use of PLA in food or feed. However, in that study, asexual development was not suppressed, and it has also 
been reported that there is a strong correlation between sporulation and toxin synthesis in molds [36]. The introduction of PLA-producing LAB strains to food and feed would hypothetically reduce sporulation, and, thus, may also suppress rather than stimulate mycotoxin synthesis. However, this hypothesis is likely dependent on mold species and possibly the target food, and requires further investigation. Compared to other preservatives, a relative high concentration of PLA is required to prevent growth. However, even at concentrations lower than the MIC, reductions in growth, and, for some molds, also the number of spores produced, would contribute to the overall antifungal activity, particularly in combination with other small molecules produced by LAB. This assumption is supported by a previous observation the in sourdough bread started with the PLA-producing L. plantarum strain $21 \mathrm{~B}$, growth of $A$. niger was delayed for 7 days compared to bread started with $L$. brevis, which do not produce PLA [37].

Expression analysis of a few selected genes involved in conidial development and, for some, possibly also stress protection, yielded some interesting trends. The increased expression of vosA in colonies grown for 7 days with both LA and PLA is in accordance with the findings of $\mathrm{Ni}$ and $\mathrm{Yu}$ for $A$. nidulans [32]: after induction of asexual development, the expression of $\operatorname{vos} A$ was elevated. This probably reflects the vosA negative feedback on sporulation, and is active to a similar extent in both LA and PLA cultures. However, the number of produced spores in PLA cultures after 7 days was significantly lower than in LA cultures (see Figure 2B), which might suggest that $\operatorname{vos} A$ is not activated by sporulation per se but rather the age of the colony or some other factor. The delayed sporulation in PLA cultures is in keeping with the fact that $\operatorname{brlA}$ expression (governing stipe development) was not observed in PLA 3-day cultures. There were no significant differences in the expression of tppA or treB between the LA and PLA assays, indicating that the sporulation-inhibiting properties of PLA are not coupled to trehalose accumulation or breakdown.

The expression of phiA after 7 days with PLA was strongly up-regulated compared to the expression after 3 days with PLA and in the LA controls (both 3 and 7 days). In $A$. nidulans the encoded protein, PhiA, is mostly present in phialides and in the conidia, and, based on sequence similarities, is proposed to be located in the cell wall. As a consequence, the $A$. nidulans deletion mutant failed to develop normal phialides and the number of conidia was reduced [29]. Moreover, phiA was initially identified as a gene induced by the Streptomyces-produced inhibitors of V-ATPases, bafilomycin and concanamycin, in Aspergillus nidulans [26,27] and later also shown in $A$. niger [28]. It is intriguing that the small LAB-produced molecule PLA induces the same gene as the large macrolide antibiotics produced by Streptomyces sp. This may indicate that PhiA, besides its role in asexual structures, is a general stress protectant produced by the fungus in competition with antagonistic bacteria.

\section{Conclusions}

In addition to a broadened knowledge of fungal-bacterial interactions, the results derived from this study strongly support the usage of LAB strains synthesizing PLA as starter cultures in food and feed. Moreover, examining outgrowth of individual conidia on solid media has certain advantages over assays in liquid media with a large number of spores, namely, changes in radial growth rate and colony morphology can be observed. The dispersal of many common spoilage molds is dependent on airborne spores; therefore, inhibited sporulation induced by PLA potentially reduces fungal contamination, in addition to inhibiting growth.

\section{Abbreviations}

PLA: Phenyllactic acid; LAB: Lactic acid bacteria; LA: Lactic acid; MEA: Malt extract agar; ACM: Aspergillus complete medium; MIC: Minimal inhibitory concentration; BA: Benzoic acid.

\section{Competing interests}

The authors declare the absence of competing interests.

\section{Authors' contributions}

ÅS and SB contributed equally to the laboratorial work. EB also contributed to the laboratorial work. Ås and PM conceived and designed the study and wrote the manuscript. All authors read and approved the final manuscript.

\section{Acknowledgements}

The authors thank Associate Professor Stefan Roos and Dr Su-lin Leong for valuable comments on the manuscript. Carl Tryggers Stiftelse (grant nr CTS08255), the Swedish research council Formas (grant nr 2008-1038), and the Italian Ministry of Instruction and Research financed this research.

\section{Author details}

${ }^{1}$ Uppsala BioCenter, Department of Microbiology, Swedish University of Agricultural Sciences, P.O. Box 7025, Uppsala SE-750 07, Sweden. ${ }^{2}$ Present address: Department of Agricultural and Food Sciences, University of Modena and Reggio Emilia, Via Amendola, Reggio Emilia 2-42100, Italy.

Received: 7 June 2013 Accepted: 8 November 2013 Published: 14 November 2013

\section{References}

1. Pitt Jl, Hocking AD: Fungi and food spoilage, 3rd edition. London, UK: Springer; 2009.

2. Leroy F, De Vuyst L: Lactic acid bacteria as functional starter cultures for the food fermentation industry. Trends Food Sci Technol 2004, 15:67-78.

3. Gobbetti M: The sourdough microflora: interactions of lactic acid bacteria and yeasts. Trends Food Sci Technol 1998, 9:267-274

4. Lindgren SE, Dobrogosz WJ: Antagonistic activities of lactic-acid bacteria in food and feed fermentations. FEMS Microbiol Rev 1990, 87:149-163.

5. Broberg A, Jacobsson K, Strom K, Schnurer J: Metabolite profiles of lactic acid bacteria in grass silage. Appl Environ Microbiol 2007, 73:5547-5552.

6. Dalie DKD, Deschamps AM, Richard-Forget F: Lactic acid bacteria - potential for control of mould growth and mycotoxins: a review. Food Control 2010, 21:370-380.

7. Schnürer J, Magnusson J: Antifungal lactic acid bacteria as biopreservatives. Trends Food Sci Technol 2005, 16:70-78.

8. Dal Bello F, Clarke Cl, Ryan LAM, Ulmer H, Schober TJ, Strom K, Sjogren J, van Sinderen D, Schnurer J, Arendt EK: Improvement of the quality and 
shelf life of wheat bread by fermentation with the antifungal strain Lactobacillus plantarum FST 1.7. J Cereal Sci 2007, 45:309-318.

9. Lavermicocca P, Valerio F, Visconti A: Antifungal activity of phenyllactic acid against molds isolated from bakery products. Appl Environ Microbiol 2003, 69:634-640.

10. Ström K, Sjogren J, Broberg A, Schnürer J: Lactobacillus plantarum MiLAB 393 produces the antifungal cyclic dipeptides cyclo(L-Phe-L-Pro) and cyclo(L-Phe-trans-4- OH-L-Pro) and 3-phenyllactic acid. Appl Environ Microbiol 2002, 68:4322-4327.

11. Ström K, Schnürer J, Melin P: Co-cultivation of antifungal Lactobacillus plantarum MiLAB 393 and Aspergillus nidulans, evaluation of effects on fungal growth and protein expression. FEMS Microbiol Lett 2005, 246:119-124.

12. Dieuleveux $V$, Lemarinier $S$, Gueguen $M$ : Antimicrobial spectrum and target site of D-3-phenyllactic acid. Int J Food Microbiol 1998, 40:177-183.

13. Brunhuber NMW, Thoden JB, Blanchard JS, Vanhooke JL: Rhodococcus L-phenylalanine dehydrogenase: kinetics, mechanism, and structural basis for catalytic specifity. Biochemistry 2000, 39:9174-9187.

14. Valerio F, Lavermicocca P, Pascale M, Visconti A: Production of phenyllactic acid by lactic acid bacteria: an approach to the selection of strains contributing to food quality and preservation. FEMS Microbiol Lett 2004, 233:289-295.

15. Gobbetti M, Corsetti A, Rossi J: The sourdough microflora. Interactions between lactic acid bacteria and yeasts: metabolism of carbohydrates. Appl Microbiol Biotechnol 1994, 41:456-460.

16. Oberdoerster J, Guizzetti M, Costa LG: Effect of phenylalanine and its metabolites on the proliferation and viability of neuronal and astroglial cells: possible relevance in maternal phenylketonuria. J Pharmacol Exp Ther 2000, 295:295-301.

17. Bauer BE, Rossington D, Mollapour M, Mamnun Y, Kuchler K, Piper PW: Weak organic acid stress inhibits aromatic amino acid uptake by yeast, causing a strong influence of amino acid auxotrophies on the phenotypes of membrane transporter mutants. Eur J Biochem 2003, 270:3189-3195.

18. Hunter DR, Segel IH: Effect of weak acids om amino acid transport by Penicillium chrysogenum: evidence for a proton or charge gradient as the driving force. J Bacteriol 1973, 113:1184-1192.

19. Sheu CW, Konings WN, Freese E: Effects of acetate and other short-chain fatty acids on sugar and amino acid uptake of Bacillus subtilis. J Bacteriol 1972, 111:525-530

20. Melin P, Stratford M, Plumridge A, Archer DB: Auxotrophy for uridine increases the sensitivity of Aspergillus niger to weak-acid preservatives. Microbiology 2008, 154:1251-1257.

21. Plumridge A, Hesse SJA, Watson AJ, Lowe KC, Stratford M, Archer DB: The weak acid preservative sorbic acid inhibits conidial germination and mycelial growth of Aspergillus niger through intracellular acidification. Appl Environ Microbiol 2004, 70:3506-3511.

22. Pel HJ, de Winde JH, Archer DB, Dyer PS, Hofmann G, Schaap PJ, Turner G, de Vries RP, Albang R, Albermann K, et al: Genome sequencing and analysis of the versatile cell factory Aspergillus niger CBS 513.88. Nat Biotechnol 2007, 25:221-231.

23. Bos CJ, Debets AJM, Swart K, Huybers A, Kobus G, Slakhorst SM: Genetic-analysis and the construction of master strains for assignment of genes to 6 linkage groups in Aspergillus niger. Curr Genet 1988, 14:437-443.

24. Vanhartingsveldt W, Mattern IE, Vanzeijl CMJ, Pouwels PH, Vandenhondel C: Development of a homologous transformation system for Aspergillus niger based on the pyrG gene. Mol Gen Genet 1987, 206:71-75.

25. Svanstrom A, Melin P: Intracellular trehalase activity is required for development, germination and heat-stress resistance of Aspergillus niger conidia. Res Microbiol 2013, 164:91-99.

26. Melin P, Schnürer J, Wagner EGH: Changes in Aspergillus nidulans gene expression induced by bafilomycin, a Streptomyces-produced antibiotic. Microbiology 1999, 145:1115-1122.

27. Melin P, Schnürer J, Wagner EGH: Proteome analysis of Aspergillus nidulans reveals proteins associated with the response to the antibiotic concanamycin A, produced by Streptomyces species. Mol Genet Genomics 2002, 267:695-702.

28. Schachtschabel D, Arentshorst M, Lagendijk EL, Ram AFJ: Vacuolar H+-ATPase plays a key role in cell wall biosynthesis of Aspergillus niger. Fungal Genet Biol 2012, 49:284-293.

29. Melin P, Schnurer J, Wagner EGH: Characterization of phiA, a gene essential for phialide development in Aspergillus nidulans. Fungal Genet Biol 2003, 40:234-241.
30. Gancedo C, Flores C-L: The importance of a functional trehalose biosynthetic pathway for the life of yeasts and fungi. FEMS Yeast Res 2004, 4:351-359.

31. Puttikamonkul S, Willger SD, Grahl N, Perfect JR, Movahed N, Bothner B, Park S, Paderu P, Perlin DS, Cramer RA Jr: Trehalose 6-phosphate phosphatase is required for cell wall integrity and fungal virulence but not trehalose biosynthesis in the human fungal pathogen Aspergillus fumigatus. Mol Microbiol 2010, 77:891-911.

32. $\mathrm{Ni} \mathrm{M}, \mathrm{Yu} \mathrm{J}-\mathrm{H}$ : A novel regulator couples sporogenesis and trehalose biogenesis in Aspergillus nidulans. PLoS One 2007, 2:e970.

33. Adams TH, Wieser JK, Yu JH: Asexual sporulation in Aspergillus nidulans. Microbiol Mol Biol Rev 1998, 62:35.

34. Samson RA, Hoekstra ES, Frisvad JC, Filtenborg O: Introduction to food and airborne fungi. 6th edition. Centralbureau voor Schimmelcultures: Utrecht, The Netherlands; 2000

35. Schmidt-Heydt M, Baxter E, Geisen R, Magan N: Physiological relationship between food preservatives, environmental factors, ochratoxin and otapksPV gene expression by Penicillium verrucosum. Int J Food Microbiol 2007, 119:277-283

36. Calvo AM, Wilson RA, Bok JW, Keller NP: Relationship between secondary metabolism and fungal development. Microbiol Mol Biol Rev 2002, 66:447-459.

37. Lavermicocca P, Valerio F, Evidente A, Lazzaroni S, Corsetti A, Gobbetti M: Purification and characterization of novel antifungal compounds from the sourdough Lactobacillus plantarum strain 21B. Appl Environ Microbiol 2000, 66(9):4084-4090.

doi:10.1186/1756-0500-6-464

Cite this article as: Svanström et al:: The lactic acid bacteria metabolite phenyllactic acid inhibits both radial growth and sporulation of filamentous fungi. BMC Research Notes 2013 6:464.

\section{Submit your next manuscript to BioMed Central and take full advantage of:}

- Convenient online submission

- Thorough peer review

- No space constraints or color figure charges

- Immediate publication on acceptance

- Inclusion in PubMed, CAS, Scopus and Google Scholar

- Research which is freely available for redistribution

Submit your manuscript at www.biomedcentral.com/submit
C Biomed Central 